\title{
GROUPTHEORETICAL CHARACTERIZATION OF PROJECTIVE SPACE AND CONFORMAL SPACE
}

\author{
MINORU KURITA
}

In this paper we characterize a projective space and a conformal space, namely a space of inversive geometry of point and sphere, from the standpoint of a homogeneous space. In such spaces a covariant differential of a vectorfield is not a vector, contrary to the case stated in my previous paper "On the vector in homogeneous spaces." (This journal vol. 5. This paper will be referred to as [1] below.) But when we restrict a rotation about a point to a certain subgroup of the full rotation group, we get a covariant differential which is also a vector, and this situation holds good in a general homogeneous space. If the fundamental group $\&$ of a homogeneous space is generated by the full group of rotation about a point and a commutative subgroup of $\mathbb{B}$ which operates simply transitively on our space, a translation of a vector can be defined smoothly on the whole space. We call such a space a space of projective type, a projective space and a conformal space being such. We characterize among the homogeneous spaces of projective type a projective space and conformal space by certain properties. In particular a conformal space of dimension greater than 2 can be characterized as a homogeneous space of projective type whose linear group of isotropy is conformal.

\section{Projective space and conformal space}

1.1 Let $\&$ be a group of projective transformations of $n$-dimensional projective space, and $A_{0}, A_{1}, \ldots, A_{n}$ be a set of analytic points in the space, which we can take as a frame of our space. Then putting $d A_{i}=\sum_{j=0}^{n} \omega_{i j} A_{j}$ $\left(\sum_{i=0}^{n} \omega_{l i}=0, i=0,1, \ldots, n\right)$ we have as structure equations

$$
\begin{array}{ll}
d \omega_{0 i}=\left[\omega_{00} \omega_{0 i}\right]+\sum_{k}\left[\omega_{0 k} \omega_{k i}\right], & d \omega_{00}=\sum_{i}\left[\omega_{0 i} \omega_{i 0}\right] \\
d \omega_{i 0}=\left[\omega_{i 0} \omega_{00}\right]+\sum_{k}\left[\omega_{i k} \omega_{k 0}\right], & d \omega_{i j}=\left[\omega_{i 0} \omega_{0 j}\right]+\sum_{k}\left[\omega_{i k} \omega_{k j}\right] .
\end{array}
$$

Denoting by $A$ an $n \times 1$ matrix with $A_{0}, A_{1}, \ldots, A_{n}$ as its coefficients we can write $d A_{i}=\sum_{j} \omega_{i j} A_{j}$ in the form $d A=\Omega A, \Omega=\left(\omega_{i j}\right)$. We take any projective transformation $P$ which fixes the point $A_{0}$ and whose determinant is \pm 1 and Received June 16, 1952. 
put

$$
\bar{A}=\left(\begin{array}{c}
\bar{A}_{0} \\
\bar{A}_{1} \\
\vdots \\
\dot{\bar{A}}_{n}
\end{array}\right)=P A, \quad P=\left(\begin{array}{cc}
\rho & 0 \\
p & P_{0}
\end{array}\right), \quad P^{-1}=\left(\begin{array}{cc}
1 & 0 \\
\rho & 0 \\
q & P_{0}^{-1}
\end{array}\right)
$$

where $p$ and $q$ are $n \times 1$ matrices, $P_{0}$ is an $n \times n$ matrix and moreover $p+\rho P_{0} q$ $=0, \rho\left|P_{0}\right|= \pm 1$. We denote the set of all such transformations by $\mathscr{S}_{2}$. Putting $d \bar{A}=\bar{\Omega} \bar{A}$ we get

$$
\bar{\Omega}=P \Omega P^{-1}+d P P^{-1}
$$

When we put

$$
\Omega=\left(\begin{array}{cc}
\omega_{00} & \omega_{1} \\
\omega_{2} & \Omega_{0}
\end{array}\right), \quad \bar{\Omega}=\left(\begin{array}{cc}
\bar{\omega}_{00} & \bar{\omega}_{1} \\
\bar{\omega}_{2} & \bar{\Omega}_{0}
\end{array}\right)
$$

where $\omega_{1}$ is a $1 \times n$-matrix and $\omega_{2}$ is an $n \times 1$ matrix, we get

$$
\begin{aligned}
& \bar{\omega}_{1}=\rho \omega_{1} P_{0}^{-1} \\
& \bar{\omega}_{00}=\rho \omega_{1} q+\omega_{00}+\frac{d \rho}{\rho}, \quad \bar{\Omega}_{0}=p \omega_{1} P_{0}^{-1}+P_{0} \Omega_{0} P_{0}^{-1}+d P_{0} P_{0}^{-1} \\
& \bar{\omega}_{2}=\frac{1}{\rho}\left(p \omega_{00}+P_{0} \omega_{2}\right)+p \omega_{1} q+P_{0} \Omega_{0} q+\frac{d p}{\rho}+d P_{1} q .
\end{aligned}
$$

(1.2) is the transformation of principal relative components by a linear group of isotropy in the projective space with points as its elements. We define a vector in such a homogeneous space by a set of numbers $v=\left(v_{1}, \ldots, v_{n}\right)$ which is transformed in such a way that

$$
\bar{v}=\rho v P_{0}^{-1}
$$

by a projective transfomation which fixes $A_{0}$. We take a vectorfield which is differentiable. Then we get by (1.2) (1.3) (1.4)

$$
d \bar{v}-\bar{\omega}_{00} \bar{v}+\bar{v} \bar{\Omega}_{0}+\rho \omega_{1} q \bar{v}=\rho\left(d v-\omega_{00} v+v \Omega_{0}-\rho v q \omega_{1}\right) P_{0}^{-1} .
$$

Hence the term in the bracket of the second side does not give a vector. But if we restrict the rotation about a point $A_{0}$ to the element of its subgroup satisfying the relation $q=0$ we get

$$
d \bar{v}-\bar{\omega}_{00} \bar{v}+\bar{v} \bar{\Omega}_{0}=\rho\left(d v-\omega_{00} v+v \Omega_{0}\right) P_{0}^{-1} .
$$

For such elements we have on account of the relation $q=0$

$$
\omega_{0 i}=0, \quad \omega_{i 0}=0 .
$$

If we add the relation $\rho=1$ we have

$$
\omega_{0 i}=0, \quad \omega_{00}=0, \quad \omega_{i 0}=0 .
$$


Now the integral manifold of the differential equation

$$
\omega_{i 0}=0
$$

containing an identity of $\$ S$ forms a subgroup and if we restrict the rotation to the elements of such a subgroup we get an affine space of dimension $\boldsymbol{n}$. By such a restriction of the rotation a covariant differential of a vector $v$ which is defined by $D v=d v-\omega_{00} v+v \Omega_{0}$ is also a vector and the geodesic can be defined by the curve get by solving

$$
\frac{d}{d \sigma}\left(\frac{\omega_{1}}{d \sigma}\right)-\frac{\omega_{00}}{d \sigma} \frac{\omega_{1}}{d \sigma}+\frac{\omega_{1}}{d \sigma} \frac{\Omega_{0}}{d \sigma}=0 \quad\left(\omega_{i 0}=0\right)
$$

which also can be obtained by solving $\omega_{1}=c_{1} d \sigma, \omega_{00}=0, \omega_{i 0}=0, \Omega_{0}=0$ and is nothing but a straight line. Next for the elements of $\$$ satisfying $\rho=1$ and

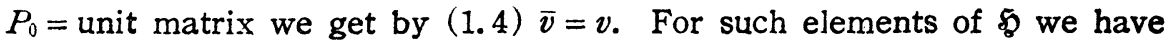

$$
\omega_{0 i}=0, \quad \omega_{0 s}=0, \quad \omega_{i j}=0 .
$$

These are the guiding ideas for our investigation. When we put $\omega_{i}=\omega_{0 i}, \pi_{i j}$ $=\omega_{i j}-\delta_{i j} \omega_{00}$ we get instead of (1.1) the following

$$
\left\{\begin{array}{l}
d \omega_{i}=\sum_{j}\left[\omega_{j} \pi_{j i}\right], \quad d \omega_{00}=\sum_{i}\left[\omega_{i} \omega_{i 0}\right], \quad d \omega_{i 0}=\sum_{j}\left[\pi_{i j} \omega_{j 0}\right] \\
d \pi_{i j}=\sum_{k}\left[\pi_{i k} \pi_{k j}\right]+\left[\omega_{i 0} \omega_{j}\right] \quad(i \neq j) \\
d \pi_{i i}=\sum_{k}\left[\pi_{i k \pi k i}\right]+\left[\omega_{i 0} \omega_{i}\right]-\sum_{j}\left[\omega_{j} \omega_{j 0}\right] .
\end{array}\right.
$$

1.2 An analogous situation holds in the conformal space with points as its elements. We take two points $A_{0}, A_{\infty}$ and $n$ spheres $A_{1}, A_{2}, \ldots, A_{n}$ such that

$$
\begin{aligned}
& \left(A_{0} A_{0}\right)=0, \quad\left(A_{0} A_{i}\right)=0, \quad\left(A_{0} A_{\infty}\right)=-1, \\
& \left(A_{i} A_{j}\right)=\delta_{i j}, \quad\left(A_{i} A_{\infty}\right)=0, \quad\left(A_{\infty} A_{\infty}\right)=0
\end{aligned}
$$

and take these as a frame of our space. Then putting $d A_{i}=\sum_{j=0}^{\infty} \omega_{i j} A_{j}(i, j=0$, $1, \ldots, n, \infty)$ and $\omega_{0 i}=\omega_{i}, \pi_{i j}=\omega_{i j}-\delta_{i j} \omega_{00}(i, j=1, \ldots, n)$ we get

$$
\begin{cases}d \omega_{i}=\sum_{j}\left[\omega_{j} \pi_{j i}\right], \quad d \omega_{00}=\sum_{i}\left[\omega_{i} \omega_{i 0}\right], & d \omega_{i 0}=\sum_{j}\left[\pi_{i j} \omega_{j 0}\right] \\ d \pi_{i j}=\sum_{k}\left[\pi_{i k} \pi_{k j}\right]+\left[\omega_{i 0} \omega_{j}\right]+\left[\omega_{i} \omega_{j 0}\right] & (i \neq j) \\ \pi_{i j}=-\pi_{j i}(i \neq j), \quad \pi_{i i}=-\omega_{00} . & \end{cases}
$$

As to (1.6) (1.7) (1.8) the assertion concerning the projective space holds good in the conformal space too.

Thus we get the following schema of subgroups for these two spaces. In this schema $\omega_{i}=0, \pi_{i j}=0$, for example, means a subgroup satisfying these conditions. 


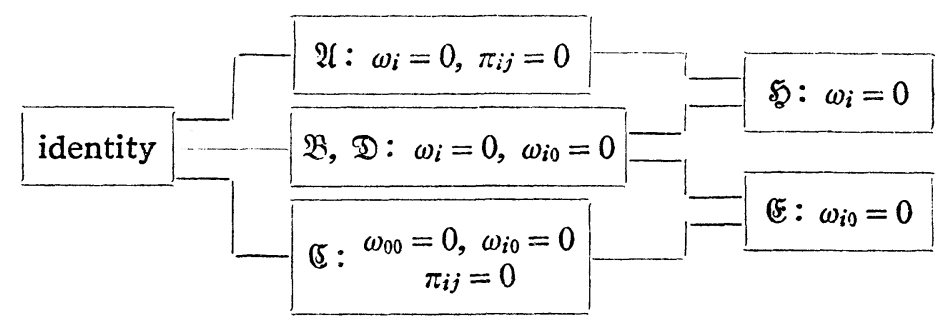

\section{Homogeneous space of projective type}

2.1 Let $\&$ be $r$-parametric Lie group which operates transitively on the space of dimension $n$ and let an element of $\&$ be given by $S_{a}: x_{i}^{\prime}=f_{i}\left(x_{1}, \ldots\right.$, $\left.x_{n} ; a_{1}, \ldots, a_{r}\right)(i=1, \ldots, n)$. Let the subgroup $\mathscr{S}$ of $\&$ which fixes a certain fixed point of the $n$-dimensional space be closed and connected. We denote by $\omega_{1}, \ldots, \omega_{n}$ principal relative components of our homogeneous space $\$ / \mathfrak{S}$ and by $\omega_{n+1}, \ldots, \omega_{r}$ secondary relative components. We use indices in the following manner throughout the discussion

$$
\begin{gathered}
i, j, k, l, h=1,2, \ldots, n \quad p, q, s, t, u=1,2, \ldots, r \\
\alpha, \beta, r, \delta=n+1, \ldots, r
\end{gathered}
$$

We have

$$
d \omega_{i}=\sum_{(p j)} c_{p j i}\left[\omega_{p} \omega_{j}\right], \quad d \omega_{\alpha}=\sum_{(p q)} c_{p q \alpha}\left[\omega_{p} \omega_{q}\right] .
$$

Let a frame at a point $x$ be $S_{a} R$ for which relative components are $\omega_{i}, \omega_{\alpha}$. If we rotate the frame about the point $x$ and get $S_{a} S_{t} R\left(S_{t} \in \mathscr{S}\right)$ we obtain for the relative components $\bar{\omega}_{i}, \bar{\omega}_{c t}$ of $S_{a^{\prime}}=S_{a} S_{t}$ the relations

$$
\bar{\omega}_{i}=\sum_{j} \tau_{i j} \omega_{j}, \quad \bar{\omega}_{\alpha}=\sum_{p} \tau_{\alpha p}()_{p}+\omega_{\alpha}^{(0)}
$$

where $\left(\tau_{p q}\right)=\left(\tau_{p q}(t)\right)$ is an element of a linear adjoint group corresponding to $S_{t}$ and $\omega_{\alpha}^{(0)}$ 's are the relative components of $S_{t}$. As $\mathscr{S}$ is connected we have

$$
\begin{aligned}
& c_{\alpha \beta i}=0, \quad \tau_{i \alpha}=0 \\
& d \tau_{p q}=\sum_{\alpha u} c_{\alpha u p} \omega_{\alpha}^{(0)} \tau_{u q}, \quad d \tau_{i j}=\sum_{\alpha i} c_{\alpha k i} \omega_{\alpha}^{(0)} \tau_{k j} . \\
& \sum_{q} c_{s t q} \tau_{p q}=\sum_{q r} c_{q r p} \tau_{q s} \tau_{r t}, \quad \sum_{j} c_{\alpha k j} \tau_{i j}=\sum_{\beta j} c_{\beta j i} \tau_{\beta \alpha} \tau_{j k} .
\end{aligned}
$$

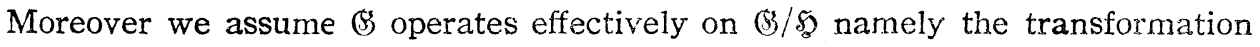
which fixes all the points of $(\mathfrak{S} / \mathscr{2}$ is nothing but the identity.

2.2 As defined in [1] 2.2 a vector in a hornogeneous space is a set of real numbers $v=\left(v_{1}, \ldots, v_{n}\right)$ corresponding to $\mathrm{S}_{a} R$ which is transformed by a frame transformation from $S_{a} R$ to $S_{a} S_{i} R$ in such a way that 


$$
\bar{v}_{i}=\sum_{j} \tau_{i j} v_{j} .
$$

Then using the notations as in [1] 2.2 we get by [1] (2.10)

$$
d \bar{v}_{i}-\sum_{\alpha k} c_{\alpha k i} \bar{\omega}_{\alpha} \bar{v}_{k}=\sum_{j} \tau_{i j}\left(d v_{j}-\sum_{\alpha k} c_{\alpha k j} \omega_{\alpha} v_{k}\right)-\sum_{\alpha k h j} c_{\alpha k i} \tau_{\alpha h} \tau_{k j} \omega_{h} v_{j} .
$$

Putting as in [1] 2.2

$$
\begin{gathered}
B_{i j h}=\sum_{\alpha} c_{\alpha i j \tau_{\alpha h},} \\
d B_{i j h}=\sum_{\gamma}\left(-\sum_{k} c_{k \gamma j} B_{i k h}+\sum_{k} c_{i \gamma k} B_{k j h}+\sum_{\alpha k} c_{\alpha i j} c_{\gamma k \alpha} \tau_{k h}\right) \omega_{\gamma}^{(0)} .
\end{gathered}
$$

If we restrict the rotations about a point $x$ to elements of $\mathscr{S}$ satisfying

$$
\omega_{i}=0, \quad \sum_{\alpha \Upsilon} c_{\alpha i j} c_{\Upsilon k_{\alpha}} \omega_{\Upsilon}=0
$$

we get $B_{i j h}=0$ on account of $(2.9)$ and $B_{i j h}=0$ for $S_{t}=$ identity. Then we have by (2.7) that $D v_{i}=d v_{i}-\sum_{\alpha k} c_{\alpha k i} \omega_{\alpha} v_{k}$ is a component of a vector. That the integral manifold of (2.10) containing the identity transformation gives a subgroup can be verified by Frobenius's theorem in the following way.

$$
d\left(\sum_{\alpha} c_{\alpha k \hat{\delta}} \omega_{\alpha}\right)=\sum_{\alpha(i j)} c_{i j \alpha} c_{\alpha k \delta}\left[\omega_{i} \omega_{j}\right]+\sum_{\alpha \beta i} c_{\alpha k \delta} c_{\beta i \alpha}\left[\omega_{\beta} \omega_{i}\right]+\sum_{\alpha(\beta \gamma)} c_{\alpha k \delta} c_{\beta \gamma \alpha}\left[\omega_{\beta} \omega_{\mathrm{r}}\right]
$$

$$
\begin{aligned}
& \sum_{\alpha} c_{\alpha k \delta} c_{\beta \top \alpha}=-\sum_{i} c_{\gamma k i} c_{i \beta \delta}-\sum_{\alpha} c_{\gamma k \alpha} c_{\alpha, \beta}-\sum_{i} c_{k_{i} i} c_{i \top \delta}-\sum_{\alpha} c_{k \beta \alpha} c_{\alpha \gamma \delta} \\
& \sum_{\delta} c_{\alpha \beta \delta} c_{\tilde{\delta} j h}=-\sum_{l} c_{\beta j l} c_{l \alpha h}-\sum_{l} c_{j \alpha l} c_{l} h \text {. }
\end{aligned}
$$

Hence $d \omega_{i}, d\left(\sum_{\alpha \delta} c_{\delta j h} c_{\alpha k \delta} \omega_{\alpha}\right)$ vanish under the relation (2.10) and the integral manifold of (2.10) containing an identity forms a subgroup. We denote this by $D$ in correspondence with that in the schema (1.12). It can also be verified by (2.11) and (2.12) that

$$
\omega_{i}=0, \quad \sum_{\gamma} c_{r k \alpha} \omega_{\gamma}=0
$$

determines a subgroup. But the equation

$$
\sum_{r} c_{r k \alpha} \omega_{r}=0
$$

is, in general, not completely integrable. It is so when and only when

$$
\sum_{\alpha} c_{i j \alpha} c_{\alpha k \delta}=0
$$

as is evident from the consideration of (2.11),

Thus we get the following theorem

THEOREM 2.1 Let $\left(v_{1}, \ldots, v_{n}\right)$ be components of a vector of a differentiable vectorfield on the homogeneous space corresponding to the frame $S_{a} S_{t} R\left(S_{t}\right.$ 
$\in \mathfrak{E})$. Then $D v_{i}=d v_{i}-\sum_{\alpha k} c_{\alpha k i} \omega_{\alpha} v_{k}$ is not in general a component of a vector. $B u t$ if we restrict $S_{t}$ to the elements of a subgroup satisfying the relations (2.10) Dvi forms a component of a vector.

ThEOREM 2.2 The equation (2.13) determines a subgoup. Let it be $\mathfrak{B}$. Even if we choose $S_{a}$ suitably for each point of our homogeneous space corresponding to $S_{a}$, a set of all $S_{a} \mathfrak{B}$ does not constitute a homogeneous space. It is so when and only when (2.15) holds.

2. 3 Now we consider the space in which (2.15) holds. In such a space we can define a parallel translation of a vector by restricting the frames at each point to $S_{a} B R$, mentioned to in theorem 2.2. Then the translation of a vector can be defined in our space by the equation

$$
D v_{i}=d v_{i}-\sum_{\alpha j} c_{\alpha j i} \omega_{\alpha} v_{j}=0
$$

and this definition is intrinsic in the sense that the equation does not depend on the choice of the frame $S_{a} S_{t} R\left(S_{t} \in \mathcal{B}\right)$. When we translate a vector from one point to another the resulting vector depends in general on the path joining these two points. It is independent when and only when the equation (2.16) is completely integrable. By Frobenius's theorem this condition can be paraphrazed into

$$
\sum_{\alpha} c_{i j \alpha \alpha} c_{\alpha k h}=0
$$

as was shown in [1] 3.2. Here we state a lemma.

Lemma. A set of all equations $\sum_{\alpha} e_{\alpha} c_{\alpha k r}=0, \sum_{\alpha} e_{\alpha} c_{\alpha k h}=0$ leads to $e_{\alpha}=0$ ( $\alpha$ $=n+1, \ldots, r)$.

Proof. Let $X_{i}$ and $X_{\alpha}$ be infinitesimal operators of our group $\mathbb{B}$ corresponding to relative components $\omega_{i}$ and $\omega_{a}$. Then the set of all operators $\sum_{\alpha} e_{\alpha} X_{\alpha}$ such that $\sum_{\alpha} e_{\alpha} c_{\alpha k \gamma}=0, \sum_{\alpha} e_{\alpha} c_{\alpha k h}=0$ generates a subgroup $\mathscr{S}_{0}$ of $\mathscr{S}_{\mathcal{2}}$ which commutes with any one-parametric subgroup generated by $\sum_{i} a_{i} X_{i}$ and any

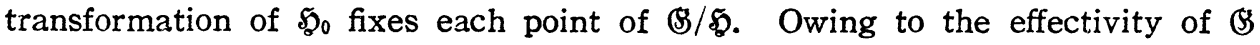
stated at the end of $2.1 \mathscr{F}_{0}$ reduces to an identity and hence $e_{\alpha}=0$. That the set of all $\sum_{\alpha} e_{\alpha} X_{\alpha}$ above stated forms an infinitesimal group and commutes with $\sum_{i} a_{i} X_{i}$ can be verified as follows.

$$
\begin{aligned}
\left(\sum_{\alpha} e_{\alpha} X_{\alpha}, \sum_{\beta} e_{\beta} X_{\beta}\right) & =\sum_{\alpha \beta \gamma} e_{\alpha} e_{\beta} c_{\alpha \beta \gamma} X_{\gamma} \\
\sum_{\gamma}\left(\sum_{\alpha \beta} e_{\alpha} e_{\beta} c_{\alpha \beta \gamma}\right) c_{\gamma k \delta} & =\sum_{\alpha \beta} e_{\alpha} e_{\beta}\left(\sum_{\gamma} c_{\alpha \beta \gamma} c_{\gamma k \delta}\right) \\
& =\sum_{\alpha \beta} e_{\alpha} e_{\beta}\left(-\sum_{i} c_{\beta k i} c_{i \alpha \delta}-\sum_{\gamma} c_{\beta k T} c_{\gamma \alpha \delta}-\sum_{i} c_{k \varangle i} c_{i \beta \delta}-\sum_{\gamma} c_{k \alpha \top} c_{\gamma \beta \delta}\right)=0
\end{aligned}
$$




$$
\begin{aligned}
& \sum_{\gamma}\left(\sum_{\alpha, \beta} e_{\alpha} e_{\beta} c_{\alpha \beta}\right) c_{\Im k i}=\sum_{\alpha, \beta} e_{\alpha} e_{\beta}\left(-\sum_{j} c_{\beta k j} c_{j \alpha i}-\sum_{j} c_{k \alpha j} c_{j \beta i}\right)=0 \\
& \left(\sum_{\alpha} e_{\alpha} X_{\alpha}, X_{i}\right)=\sum_{\alpha} e_{\alpha}\left(\sum_{j} c_{\alpha i j} X_{j}+\sum_{\beta} c_{\alpha i \beta} X_{\beta}\right)=0 .
\end{aligned}
$$

By virtue of this lemma we get from (2.15) and (2.17)

$$
c_{i j \alpha}=0 \text {. }
$$

This relation is equivalent to the condition that $X_{1}, \ldots, X_{n}$ generate a subgroup of $\$ 5$. Thus we get the following

THEOREM 2.3 In the homogeneous. space where $\sum_{\alpha} c_{i j \alpha} c_{a k \delta}=0$ holds the translation of a vector is absolute when and only when $c_{i j a}=0$ hold. This con-

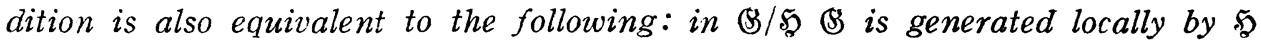
and an n-parametric subgroup of \$S.

2.4 Now we consider a space satisfying $c_{i j \alpha}=0$. Then we have

$$
d \omega_{\alpha}=\sum_{\beta k} c_{\beta k \alpha}\left[\omega_{\beta} \omega_{k}\right]+\sum_{(\beta \gamma)} c_{\beta \gamma \alpha}\left[\omega_{\beta} \omega_{r}\right]
$$

and so $\omega_{\alpha}=0$ is completely integrable. We can restrict a frame at each point

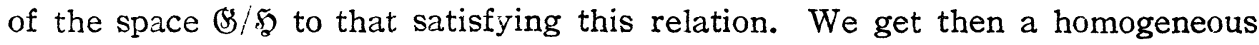
space which is transformed simply transitively, and the structure equation of such a space is given by $d \omega_{i}=\sum_{i \jmath k} c_{j k i}\left[\omega_{j} \omega_{k}\right]$. We call a transformation of our

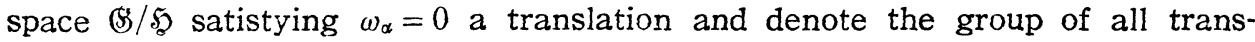
lations by $\mathfrak{I}$. The commutativity of the group $\mathfrak{I}$ can be expressed by $c_{i j k}=0$. We call the space satisfying the relations $c_{i j \alpha}=0$ and $c_{i j k}=0$ a homogeneous space of projective type. A projective space and a conformal space with points as their elements are the spaces of this type. In the space of projective type we have the relations

$$
\sum_{\alpha} c_{k \beta \alpha} c_{\alpha j i}=\sum_{\alpha} c_{j \beta \alpha} c_{\alpha k i}, \quad \sum_{\alpha} c_{k \beta \alpha} c_{\alpha j \gamma}=\sum_{\alpha} c_{j \beta \alpha} c_{\alpha k r}
$$

which can easily be verified by Jacobi's identity.

2.5 In a homogeneous space the differential equation

$$
\omega_{i}=0, \quad \sum_{\alpha} c_{\alpha i j} \omega_{\alpha}=0
$$

is completely integrable on account of the relations (2.1) and

$$
\begin{gathered}
d\left(\sum_{\alpha} c_{\alpha i j} \omega_{\alpha}\right)=\sum_{(k h ; \alpha} c_{k h \alpha} c_{\alpha i j}\left[\omega_{k} \omega_{h}\right]+\sum_{\beta k \alpha} c_{\beta k \alpha} c_{\alpha i j}\left[\omega_{\beta} \omega_{k}\right]+\sum_{(\beta \gamma) \alpha} c_{\beta \gamma \alpha} c_{\alpha i j}\left[\omega_{\beta} \omega_{r}\right] \\
\sum_{\alpha} c_{\beta \gamma \alpha} c_{\alpha i j}=-\sum_{k} c_{\gamma i k} c_{k \beta j}-\sum_{i} c_{i \beta k} c_{k r j} .
\end{gathered}
$$

In fact $d \omega_{i}=0, d\left(\sum_{\alpha} c_{\alpha i j} \omega_{\alpha}\right)=0$ under the condition (2.20).

Thus we have a schema of subgroups in a general homogeneous space. 


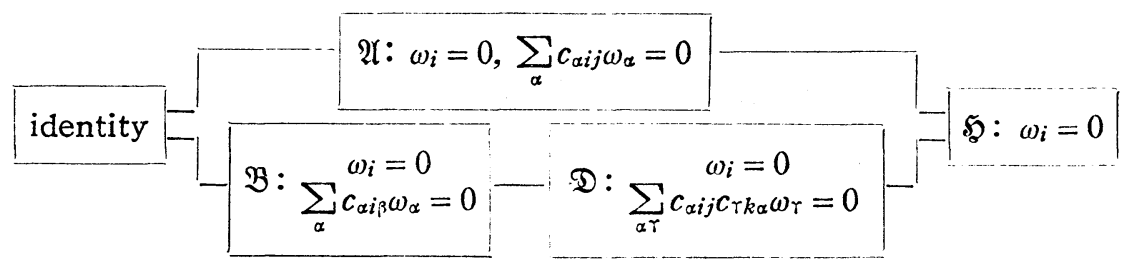

In the homogeneous space of projective type we have the following. We give attention to the correspondence with (1.12).

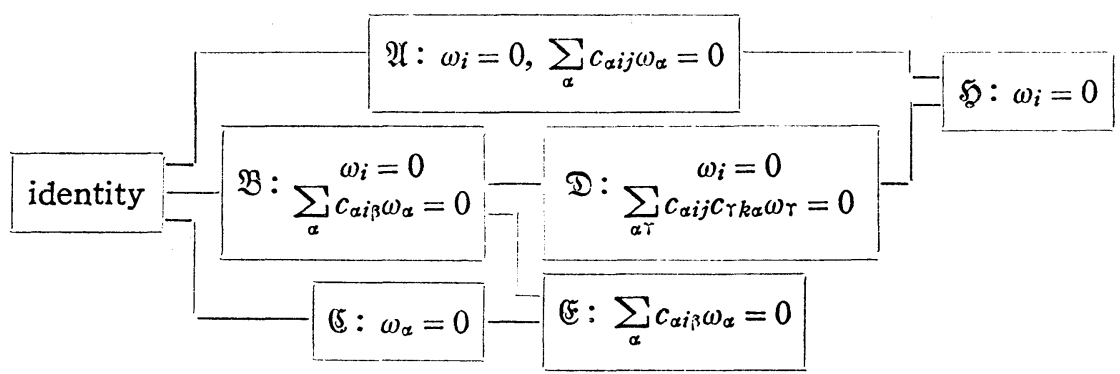

\section{Characterization of projective space}

3.1 In a homogeneous space of projective type we have

$$
d \omega_{i}=\sum_{\alpha j} c_{\alpha j i}\left[\omega_{\alpha} \omega_{j}\right]
$$

$$
d \omega_{\alpha}=\sum_{\beta k} c_{\beta k \alpha}\left[\omega_{\beta} \omega_{k}\right]+\sum_{(, 3 r)} c_{\beta r \alpha}\left[\omega_{\beta} \omega_{r}\right] \text {. }
$$

In contrast with the equation in the case of projective space (1.10) we put

$$
\pi i j=-\sum_{\alpha} c_{\alpha i j} \omega_{\alpha} \text {. }
$$

Then we have

$$
d \omega_{i}=\sum_{j}\left[\omega_{j} \pi_{j i}\right] .
$$

By virtue of (2.21) (2.19) (2.22) and the relation $c_{k h \alpha}=0$ we get

$$
d \pi_{i j}=\sum_{\alpha} c_{i \beta \alpha} c_{\alpha k j}\left[\omega_{\beta} \omega_{k}\right]+\sum_{k}\left[\pi_{i k} \pi_{k j}\right] .
$$

In a projective space we have by $(1.10)$

$$
d \pi_{i j}=\left[\omega_{i 0} \omega_{j}\right]+\sum_{l k}\left[\pi_{i k \pi_{k} j}\right] \quad(i \neq j) .
$$

So if there exist such $A_{i \beta}$ that

$$
\sum_{\alpha} c_{i \beta \alpha} c_{\alpha k j}=\sum_{\alpha} c_{k \beta \alpha} c_{\alpha i j}=A_{i \beta} \delta_{k j} \quad(i \neq j)
$$

we obtain (3.6) by putting 


$$
\omega_{i 0}=\sum_{\beta} A_{i_{\beta}} \omega_{\beta} .
$$

(3.7) being assumed we get from (3.8)

$$
d \omega_{i 0}=\sum_{\beta} A_{i \beta}\left(\sum_{\gamma k} c_{\gamma k \beta}\left[\omega_{\gamma} \omega_{k}\right]+\sum_{(\gamma \delta)} c_{\gamma \delta \beta}\left[\omega_{\gamma} \omega_{\delta}\right]\right) .
$$

In order that this reduces to

$$
d \omega_{i 0}=\sum_{k}\left[\pi_{i k} \omega_{k 0}\right]
$$

with $\omega_{i 0}, \pi_{i j}$ defined by (3.8) and (3.3) it is sufficient that we have the relations

$$
\sum_{\beta} A_{i_{\beta}} C_{\Upsilon k \beta}=0
$$

$$
\sum_{\beta} A_{i \beta} c_{\gamma \delta \beta}=-\sum_{k} c_{\gamma i k} A_{k \delta}+\sum_{k} c_{\delta i k} A_{k \gamma}
$$

Next we consider the condition in order that

$$
d \pi_{i i}=\sum_{k}\left[\pi_{i k} \pi_{k i}\right]+\left[\omega_{i 0} \omega_{i}\right]-\sum_{j}\left[\omega_{j} \omega_{j 0}\right]
$$

holds. By (3.3) and (3.7) we have

$$
\begin{aligned}
d \pi_{i i}= & -\sum_{\alpha} c_{\alpha i i}\left(\sum_{\beta k} c_{\beta k \alpha}\left[\omega_{\beta} \omega_{k}\right]+\sum_{(\beta r)} c_{\beta r \alpha}\left[\omega_{\beta} \omega_{r}\right]\right) \\
= & \sum_{k \beta} A_{k \beta}\left[\omega_{\beta} \omega_{k}\right]-\sum_{\beta} A_{i \beta}\left[\omega_{\beta} \omega_{i}\right]-\sum_{\alpha \beta} c_{\alpha i i} c_{\beta i \alpha}\left[\omega_{\beta} \omega_{i}\right] \\
& -\sum_{\alpha(\beta r)} c_{\alpha i i} c_{\beta \gamma \alpha}\left[\omega_{\beta} \omega_{r}\right] .
\end{aligned}
$$

and by (3.3) and (3.8) we have

$$
\begin{aligned}
\sum_{k}\left[\pi_{i k} \pi k_{i}\right] & +\left[\omega_{i 0} \omega_{i}\right]-\sum_{J}\left[\omega_{j} \omega_{j 0}\right] \\
& =\sum_{k}\left[\sum_{\alpha} c_{\alpha i k} \omega_{\alpha}, \sum_{\beta} c_{\beta k i} \omega_{\beta}\right]+\sum_{\beta} A_{i \beta}\left[\omega_{\beta} \omega_{i}\right]-\sum_{\beta i} A_{i \beta}\left[\omega_{i} \omega_{\beta}\right] .
\end{aligned}
$$

That the coefficients of $\left[\omega_{\beta} \omega_{i}\right]$ in (3.14) and (3.15) are the same can be verified by the Jacobi's identity $(2.22)$. As for the coefficients of $\left[\omega_{\beta} \omega_{i}\right]$ it is sufficient that

$$
\sum_{\alpha} c_{i \beta \alpha} c_{\alpha i i}=2 A_{i \beta}
$$

holds. Thus we get (3.7) (3.11) (3.12) (3.16) as sufficient conditions in order that (1.10) except $d \omega_{00}=\sum_{i}\left[\omega_{i} \omega_{i 0}\right]$ holds for $\pi_{i j}, \pi_{i 0}$ given by (3.3) (3.8). Now we remark that (3.12) is a consequence of other relations. In fact if we take $k$ which is not equal to $i$, the dimension of the space being assumed to be greater than one, then we have $A_{i \beta}=\sum_{\alpha} c_{i \beta \alpha} c_{\alpha k k}$ and hence 
(3.17) $\sum_{\beta} A_{i \beta} c_{\curlyvee \delta \beta}=\sum_{\alpha \beta} c_{\curlyvee \delta \beta} c_{i \beta \alpha} c_{\alpha k k}$

$$
=\sum_{\alpha}\left(\sum_{j} c_{\delta i j} c_{j \curlyvee \alpha}+\sum_{\beta} c_{\delta i \beta} c_{\beta \uparrow \alpha}+\sum_{j} c_{i \curlyvee j} c_{j \delta \alpha}+\sum_{\beta} c_{i \curlyvee \beta} c_{\beta \delta \alpha}\right) c_{\alpha k k}
$$

By (3.7) (3.16) (2.19) we have

$$
\begin{aligned}
\sum_{\alpha j} c_{\delta i j} c_{j \curlyvee \alpha} c_{\alpha k k} & =\sum_{j} c_{\delta i j} A_{j \curlyvee}+c_{\delta i k} A_{k \uparrow} \\
\sum_{\alpha \beta} c_{\delta i \beta} c_{\beta \gamma \alpha} c_{\alpha k k} & =-\sum_{\beta} c_{\delta i \beta}\left(\sum_{j} c_{\curlyvee k j} c_{j \beta k}+\sum_{j} c_{k \beta j} c_{j \curlyvee k}\right) \\
& =\sum_{\beta} c_{\delta i \beta} c_{\beta k k} c_{\Upsilon k k}-\sum_{\beta} c_{\delta i \beta} c_{\beta k k} c_{\curlyvee k k}-\sum_{\beta} c_{k \delta \beta} c_{\beta i i} c_{i \curlyvee k}=c_{\Upsilon i k} A_{k \delta} .
\end{aligned}
$$

Similarly $\sum_{\alpha j} c_{i \curlyvee j} c_{j \delta \alpha} c_{\alpha k k}=-\sum_{j} c_{\gamma i j} A_{j \delta}-c_{\Upsilon i k} A_{k \delta}, \sum_{\alpha \beta} c_{i \curlyvee \beta} c_{\beta \delta \alpha} c_{\alpha k k}=-c_{\delta i k} A_{k \gamma}$. Putting these into the right side of (3.17) we get (3.12). The verification of the case $n=1$ is more easy. Thus we obtain a set of conditions

$$
\begin{gathered}
c_{i j k}=0, \quad c_{i j \alpha}=0 \\
\sum_{\alpha} c_{i \beta \alpha} c_{\alpha k j}=0 \quad(i \neq j, k \neq j) \\
\frac{1}{2} \sum_{\alpha} c_{i \beta \alpha} c_{\alpha i i}=\sum_{\alpha} c_{i \beta \alpha} c_{\alpha j j}=\sum_{\alpha} c_{j \beta \alpha} c_{\alpha i j}=A_{i \beta} \quad(i \neq j) \\
\sum_{\beta} A_{i \beta} c_{T k \beta}=0 .
\end{gathered}
$$

Under these conditions we get the structure equations of a projective space (1.10) when we put

$$
\pi_{i j}=-\sum_{\alpha} c_{\alpha i j} \omega_{\alpha}, \quad \omega_{i 0}=\sum_{\beta} A_{i \beta} \omega_{\beta}, \quad \omega_{00}=\frac{1}{n+1} \sum_{\alpha i} c_{\alpha i i} \omega_{\alpha} .
$$

The formula for $\omega_{00}$ can be verified as follows. We have by virtue of the relation $\sum_{i=0}^{n} \omega_{i i}=0$

$$
\sum_{i=1}^{n} \pi_{i i}=\sum_{i=1}^{n}\left(\omega_{i i}-\omega_{00}\right)=-(n+1) \omega_{n \rho}
$$

Hence $\omega_{00}=-\frac{1}{n+1} \sum_{\alpha i} c_{\alpha i i} \omega_{\alpha}$. That this satisfies the equation $d \omega_{00}=\sum_{i}\left[\omega_{i} \omega_{i 0}\right]$ can be verified as follows.

$$
\begin{aligned}
d \omega_{00} & =\frac{1}{n+1} \sum_{\alpha i} c_{\alpha i i} d \omega_{\alpha}=\frac{1}{n+1}\left(\sum_{\alpha i j \beta} c_{j \beta \alpha} c_{\alpha i i}\left[\omega_{j} \omega_{\beta}\right]+\sum_{\alpha(\beta \gamma) i} c_{\beta \gamma_{\alpha}} c_{\alpha i i}\left[\omega_{\beta} \omega_{r}\right]\right) \\
& =\frac{1}{n+1} \sum_{j}\left[\omega_{j}, \sum_{\alpha i \beta} c_{j \beta \alpha} c_{\alpha i i} \omega_{\beta}\right]+\frac{1}{n+1} \sum_{\alpha(\beta \gamma) i} c_{\beta \gamma \alpha} c_{\alpha i i}\left[\omega_{\beta} \omega_{r}\right] \\
& =\frac{1}{n+1} \sum_{j}\left[\omega_{j},(n+1) \sum_{\beta} A_{j \beta} \omega_{\beta}\right]-\frac{1}{n+1} \sum_{(\beta \gamma)}\left(\sum_{k i} c_{\gamma i k} c_{k \beta i}+\sum_{k i} c_{i \beta k} c_{k r i}\right)\left[\omega_{\beta} \omega_{r}\right] \\
& =\sum_{j}\left[\omega_{j} \omega_{j 0}\right] .
\end{aligned}
$$


3.2 The geometrical interpretation of the relation (I) was given in 2 . Now we will interprete (II). In the homogeneous space of projective type we restrict to the frames satisfying $\sum_{\alpha} c_{\alpha k_{\beta}} \omega_{\alpha}=0$ as was mentioned to in 2.2, then a translation of a vector is defined by $D v_{i}=d v_{i}-\sum_{\alpha k} c_{a k i} \omega_{\alpha} v_{k}=0$ and a geodesic can be defined as the curve obtained by solving

$$
\frac{d}{d \sigma}\left(\frac{\omega_{i}}{d \sigma}\right)-\sum_{\alpha k} c_{\alpha k i} \frac{\omega_{\alpha}}{d \sigma} \frac{\omega_{k}}{d \sigma}=0
$$

with a suitably chosen parameter $\sigma$. The curve can also be obtained by solving

$$
\omega_{i}=c_{i} d \sigma, \quad \omega_{a}=0 \quad\left(c_{i}=\text { const. }\right) .
$$

The discussion is analogous to that of [1] 4.1. We call the frames along the geodesic satisfying (3.18) adapted frames corresponding to the geodesic. Let these be $S_{a} R$ and operate on them infinitesimal rotations $S_{\varepsilon}$. Relative displacement of these frames $S_{a} S_{\Sigma} R$ is $\left(S_{a} S_{\varepsilon}\right)^{-1} S_{a+d a} S_{\S}=S_{\varepsilon}^{-1}\left(S_{a}^{-1} S_{a+d a}\right) S_{\varepsilon}$ and its relative components are given by $\sum_{k} \tau_{p k} c_{k} d \sigma$ where $\left(\tau_{p q}\right)$ is an element of a linear adjoint group corresponding to $S_{\S}$. Hence by (2.4) we have for an infinitesimal change of relative components corresponding to the frame transformation from $S_{a} R$ to $S_{a} S_{\varepsilon} R$

$$
\delta \omega_{i}=\sum_{\alpha k} c_{\alpha k i} e_{\alpha} c_{k} d \sigma, \quad \delta \omega_{\alpha}=\sum_{\beta h} c_{\beta h \alpha} e_{\beta} c_{h} d \sigma
$$

where $\left(0, \ldots, 0, e_{n+1}, \ldots, e_{r}\right)$ are parameters for $S_{\varepsilon}$. Thus if we neglect terms of higher order with respect to $e_{\alpha}$ we get for secondary relative components of $S_{a} S_{\varepsilon} R \omega_{\alpha}+\delta \omega_{\alpha}=\delta \omega_{\alpha}=\sum_{\beta h} c_{\beta} h_{\alpha} e_{\beta} c_{h} d \sigma$. When we attach to each point of a curve in the homogenecus space a fixed frame, we can define a translation of a vector $v$ along the curve relative to the frames by the equation

$$
D v_{i}=d v_{i}-\sum_{\alpha j} c_{\alpha j i} \omega_{\alpha} v_{j}=0
$$

although the translation thus defined is not intrinsic with respect to the curve. In this sense of parallelism $c=\left(c_{1}, \ldots, c_{n}\right)$ is parallel with respect to the frames $S_{a} R$.

Let $c=\left(c_{1}, \ldots, c_{n}\right)$ be a vector which determines the geodesic (3.18) and we assume for any geodesic and $S_{\varepsilon}$ that

$$
\left\{\begin{array}{l}
\text { "when we translate a vector } c \text { with respect to frames } S_{a} S_{\varepsilon} R \text { the di- } \\
\text { rection of } c \text { is invariant except for a second order with respect to } \\
\text { the parameters of } S_{\varepsilon} . "
\end{array}\right.
$$

Then we get (II) as follows. The property (3.19) means that 


$$
D c_{i}=d c_{i}-\sum_{\alpha k} c_{\alpha k i}\left(\omega_{\alpha}+\delta \omega_{\alpha}\right) c_{k}=-\sum_{\alpha k} c_{\alpha k i}\left(\sum_{\beta h} c_{\beta h \alpha} e_{\beta} c_{h} d \sigma\right) c_{k}
$$

is proportional to $c_{i}$. Hence we can put $\sum_{\beta \alpha k h} c_{\alpha k i} c_{\beta h \alpha} e_{\beta} c_{h} c_{k}=c_{i} P$. As $e_{3}$ is arbitrary we get

$$
\sum_{\alpha k h} c_{\alpha k i} c_{\beta h \alpha} c_{h} c_{k}=c_{i} P_{\beta} .
$$

Hence $\sum_{\alpha k h} c_{\alpha k i} c_{\beta h \alpha} c_{h} c_{k} / c_{i}=P_{\beta}$ does not depend on $i$. As $c_{i}$ is arbitrary $P_{\beta}$ must be a linear combination of $c_{1}, \ldots, c_{n}$. Hence

$$
\sum_{\alpha} c_{\beta} h_{\alpha} c_{\alpha k i}=0 \quad(h \neq i . \quad k \neq i)
$$

and so

$$
P_{3}=\sum_{\alpha} c_{\beta i \alpha} c_{\alpha i i} c_{i}+\sum_{\jmath(\neq i)}\left(\sum_{\alpha} c_{\beta i \alpha} c_{\alpha j i}+\sum_{\alpha} c_{\beta j \alpha} c_{\alpha i i}\right) c_{j}
$$

As we have $\sum_{\alpha} c_{\beta i \alpha} c_{\alpha j i}=\sum_{\alpha} c_{\beta j \alpha} c_{\alpha i i}$ by (2.19) we can put

$$
\sum_{\alpha} c_{\beta i \alpha} c_{\alpha i i}=-2 A_{i \beta}, \quad \sum_{\alpha} c_{\beta j \alpha} c_{\alpha i i}=-A_{j 3} \quad(i \neq j) .
$$

Thus we obtain (II).

3. 3 Niw we will show that if the dimension of our space is greater than one the relation (III) is a consequence of (II). We begin with the case of the dimension greater than 2. Then we can take for any $i$ and $k$ a positive integer $j$ such that $i \neq j, k \neq j$. By virtue of the relation (II) and (2.19) we get

$$
\sum_{\beta} A_{i \beta} c_{r k_{\beta}}=-\sum_{\beta \alpha} c_{\gamma k_{\beta} c_{3 i \alpha}} c_{\alpha j j}=-\sum_{i \alpha \alpha} c_{r k_{\beta} \beta} c_{3 j \alpha} c_{\alpha i j}=-\sum_{; \alpha} c_{r j \beta} c_{\beta k_{\alpha} \alpha} c_{\alpha i j}=0 .
$$

In the case of dimension 2 such $j$ as we used above can be taken except for $\sum_{\beta} A_{1, \beta} c_{i 2, \beta}$ and $\sum_{\beta} A_{2 \beta} c_{r 1 \beta}$. In this case we proceed as follows. We get by (3.22) (2.19)

$$
2 \sum_{\beta} A_{1 ;} C_{r 2 \beta}=-\sum_{\beta \alpha} c_{\gamma 2 \beta} c_{\beta 1 \alpha} c_{\alpha 11}=-\sum_{\beta \alpha} c_{r 1 \beta} C_{\beta 2 \alpha} c_{\alpha 11}=\sum_{\beta} c_{r 1 \beta} A_{2 \beta} .
$$

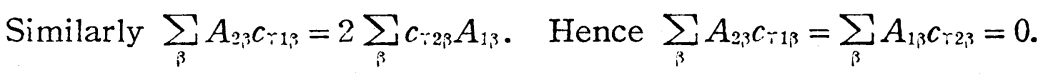

In the case of dimension one I can not give a geometric interpretation to the relation (III) namely

$$
\sum_{\beta} A_{1, \beta} c_{\Upsilon 1 \beta}=\sum_{\alpha \beta} c_{\Upsilon 1 \beta} c_{\beta 1 \alpha} c_{\alpha 11}=0
$$

Thus we get a characterization of a projective space which can be stated as follows:

THEOREM 3.1 A homogeneous space of projective type whose dimension is greater than one can be locally imbedded into the projective space of the same 
dimension preserving the group-theoretical structure if it satisfies the following condition: when we operate on all the adapted frames along any geodesic any infinitesimal rotation $S_{\S}$, the vector which is tangent to this geodesic and has constant components does not change its direction by the translation with respect to the rotated frames, except for an order greater than one.

This condition is rather complicated. It is desirable to replace it by one which is simpler. The rotation group about a point when it is imbedded into the projective space is in general a subgroup of that of the projective space.

In one-dimensional case (I) (II) are always satisfied. Hence we get the following theorem.

THEOREM 3.2 A one-dimensional homogeneous space satisfying the relation $\sum_{\alpha \beta} c_{r 13} c_{31 \alpha} c_{\alpha 11}=0$ can be imbedded into the one-dimensional projective space preserving the group-theoretical structure.

Although a projective space with points as its elements is of projective type and satisfies (3.19) the converse of theorem 3.1 is not true. A simple example shows that there exists a homogeneous space which can be imbedded into the projective space of the same dimension and yet is not of projective type. A homogeneous space which can be imbedded into the projective space of the same dimension and whose fundamental group contains a group of all translations is of projective type. In fact in such a space (1.10) holds, where $\pi_{i j}$, $\omega_{i 0}$ are linear combinations of relative components $\omega_{i}, \omega_{\alpha}$ with constant coefficients. The differential equations $\pi_{i j}=0, \omega_{i 0}=0$ are completely integrable, though $\pi_{i j}, \omega_{i v}$ are not always independent. We take frames satisfying these relations. Then owing to the assumption that the fundamental group contains a group of all translations $\omega_{i}$ 's $(i=1, \ldots, n)$ are independent even when we put $\pi_{i j}=0 . \omega_{i 0}=0$. So we can take secondary relative components $\omega_{\alpha}$ anew such that $\pi i j, \omega_{i 0}$ are linear combinations of $\omega_{\alpha}$ with constant coefficients. Then we get $c_{i j k}=0, c_{i j \alpha}=0$ and hence a space of projective type. We have in addition (3.3) (3.8), and (3.19) is sactisfied.

\section{Characterization of conformal space}

4. 1 Now we treat the case of a conformal space which is simpler than that of the projective space. In a conformal space we have by (1.11)

$$
\pi_{i i}=-\omega_{00}, \quad \pi_{\imath j}=-\pi_{j i} \quad(i \neq j) .
$$

Hence the linear group of isotropy is conformal. We take a homogeneous space of projective type and seek for the conditions in order that it can be imbedded into a conformal space. In the first we put 


$$
\pi_{i j}=-\sum_{\alpha} c_{\alpha i j} \omega_{\alpha}
$$

to get $d \omega_{i}=\sum_{j}\left[\omega_{j} \pi_{j i}\right]$ from (3.1). We assume that a linear group of isotropy of our space is conformal, namely

$$
c_{\alpha i j}=-c_{\alpha j i} \quad(i \neq j), \quad c_{\alpha i i}=c_{\alpha} .
$$

We have by (2.19) $\sum_{\alpha} c_{k \beta \alpha} c_{\alpha j i}=\sum_{\alpha} c_{j \beta \alpha} c_{\alpha k i}$. So if we put $\sum_{\alpha} c_{k_{\beta} \alpha} c_{\alpha j i}=P_{k j i}$ we have $P_{k j i}=P_{j k i}$ and by virtue of the assumption (4.2) $P_{k j i}=-P_{k i j}$ for $i \neq j$. Hence for $i \neq j \neq k$ we get

$$
P_{k j i}=-P_{k i j}=-P_{i k j}=P_{i j k}=P_{j i k}=-P_{j k i}=-P_{k j i} .
$$

Hence $P_{k j i}=0 \quad(i \neq j \neq k)$. For other cases we get

$$
P_{j j i}=-P_{j i j}=-P_{i j j}=-\sum_{\alpha} c_{i \beta \alpha} c_{\alpha j j}=-\sum_{\alpha} c_{i \beta \alpha} c_{\alpha}=-P_{i i i} \quad(i \neq j) .
$$

Hence putting $\sum_{\alpha} c_{i \beta \alpha} c_{\alpha}=A_{i \beta}$ we get

$$
\begin{aligned}
& \sum_{\alpha} c_{i \beta \alpha} c_{\alpha j k}=0 \quad(i \neq k \neq j) \\
& \sum_{\alpha} c_{i \beta \alpha} c_{\alpha j i}=-\sum_{\alpha} c_{i \beta \alpha} c_{\alpha i j}=\sum_{\alpha} c_{j \beta \alpha} c_{\alpha i i}=A_{j \beta} \quad(i \neq j) .
\end{aligned}
$$

As our space is of projective type we have by (3.5)

$$
d \pi_{i j}=\sum_{\alpha \beta k} c_{i \beta \alpha} c_{\alpha k j}\left[\omega_{\beta} \omega_{k}\right]+\sum_{k}\left[\pi_{i k} \pi_{k j}\right] .
$$

So for $i \neq j$ we obtain by (4.3)

$$
\begin{aligned}
d \pi_{i j} & =\sum_{\alpha \beta} c_{i \beta \alpha} c_{\alpha j j}\left[\omega_{\beta} \omega_{j}\right]+\sum_{\alpha, \beta} c_{i \beta \alpha} c_{\alpha i j}\left[\omega_{\beta} \omega_{i}\right]+\sum_{k}\left[\pi_{i k \pi} \pi_{k j}\right] \\
& =\sum_{\beta} A_{i \beta}\left[\omega_{\beta} \omega_{j}\right]+\sum_{\beta} A_{j \beta}\left[\omega_{\beta} \omega_{i}\right]+\sum_{k}\left[\pi_{i k} \pi_{k j}\right] .
\end{aligned}
$$

If we put

$$
\omega_{i 9}=\sum_{\beta} A_{i \beta} \omega_{\beta}
$$

we get

$$
d \pi_{i j}=\left[\omega_{i 0} \omega_{j}\right]-\left[\omega_{j 0} \omega_{i}\right]+\sum_{k}\left[\pi_{i k} \pi_{k j}\right]
$$

From (4.4) we get

$$
d \omega_{i 0}=\sum_{\beta \curlyvee k} A_{i \beta} C_{\curlyvee k_{\beta}}\left[\omega_{\curlyvee} \omega_{k}\right]+\sum_{\beta(\curlyvee \delta)} A_{i \beta} C_{\Upsilon \delta \beta}\left[\omega_{\curlyvee} \omega_{\delta}\right]
$$

and this reduces to

$$
d \omega_{i 0}=\sum_{\jmath}\left[\pi_{i j} \omega_{j 0}\right]
$$

if the relations 


$$
\begin{gathered}
\sum_{\beta} A_{i \beta} c_{\Upsilon \hat{\delta} \beta}=-\sum_{\nu} c_{\Upsilon i j} A_{j \delta}+\sum_{\partial} c_{\delta i j} A_{j \curlyvee} \\
\sum_{\beta} A_{i \beta} c_{\curlyvee k \beta}=0
\end{gathered}
$$

are satisfied. The former is a consequence of other relations as is shown in the following. We have by (4.3)

$$
\sum_{\beta} A_{i \beta} c_{\curlyvee \delta \beta}=-\sum_{\alpha \beta} c_{\gamma \delta \beta} c_{\beta i \alpha} c_{\alpha}=\sum_{j \alpha} c_{\delta i j} c_{j \gamma \alpha} c_{\alpha}+\sum_{\beta \alpha} c_{\delta i \beta} c_{\beta \zeta \alpha} c_{\alpha}+\sum_{\alpha \beta} c_{i \curlyvee \beta} c_{\beta \delta \alpha} c_{\alpha}+\sum_{j \alpha} c_{i \curlyvee j} c_{j \delta \alpha} c_{\alpha}
$$

$$
\sum_{\alpha} c_{\beta \curlyvee \alpha} c_{\alpha i i}=-\sum_{k} c_{\Upsilon i k} c_{k \beta i}-\sum_{k} c_{i \beta k} c_{k \Upsilon i}=\sum_{k} c_{\beta k i} c_{\curlyvee i k}-\sum_{k} c_{\beta k i} c_{\Upsilon i k}=0,
$$

and so we get

$$
\begin{aligned}
& \sum_{\beta} A_{i \beta} c_{r \delta \beta}=\sum_{j \alpha} c_{\delta i j} c_{j \curlyvee \alpha} c_{\alpha}+\sum_{j \alpha} c_{i \curlyvee j} c_{j \delta \alpha} c_{\alpha} \\
& =-\sum_{j \alpha} c_{\gamma i j} c_{j \delta \alpha} c_{\alpha}+\sum_{j \alpha} c_{\delta i j} c_{j \gamma \alpha} c_{\alpha}=-\sum_{\jmath} c_{r i j} A_{j \delta}+\sum_{j} c_{\tilde{\sigma} i j} A_{j \curlyvee} .
\end{aligned}
$$

As for (4.7) we can proceed as in 3.3 if $n>2$ and $i \neq k$ but if $n>1$ and $i=k$ we proceed as follows. Let $i \neq j$ then we have by (4.2) (2.19)

$$
\begin{aligned}
\sum_{\beta} A_{i \beta} c_{\Upsilon t_{3} \beta} & =\sum_{\alpha \beta} c_{\Upsilon i \beta} c_{i \beta \alpha} c_{\alpha j j}=\sum_{\alpha \beta} c_{\Upsilon i \beta} c_{j \beta \alpha} c_{\alpha i j} \\
& =-\sum_{\alpha \beta} c_{\Upsilon j \beta} c_{i \beta \alpha} c_{\alpha j i}=-\sum_{\alpha \beta} c_{\Upsilon j \beta} c_{j \beta \alpha} c_{\alpha i i}=-\sum_{\beta} A_{j \beta} c_{\Upsilon j \beta} .
\end{aligned}
$$

Hence

$$
\sum_{\beta} A_{i \beta} C_{r i \beta}+\sum_{\beta} A_{j \beta} C_{r j \beta}=0 .
$$

From the equations obtained from this by putting $j, k$ and $k, i$ in place of $i, j$ we get (4.7), and so (4.7) holds good if $n>2$. In the case $n=2$ we get only

$$
\sum_{i \alpha} c_{r 1 \beta} c_{1, \beta \alpha} c_{\alpha}+\sum_{3 \alpha} c_{r 2, \beta} c_{2,3 \alpha} c_{\alpha}=0
$$

and in order that each term reduces to 0 it is necessary and sufficient that

$$
\sum_{\alpha \beta} c_{r 1 \beta} c_{1, \beta} c_{\alpha}=\sum_{\alpha \beta} c_{r 2 \beta} c_{2 \beta} c_{\alpha} .
$$

When $n=1$ we have

$$
\sum_{\alpha \beta} c_{\Upsilon 1 \beta} c_{1 \beta \alpha} c_{\alpha 11}=0
$$

As to $\pi i i=-\omega_{00}=-\sum_{\alpha} c_{\alpha} \omega_{\alpha}$ we have

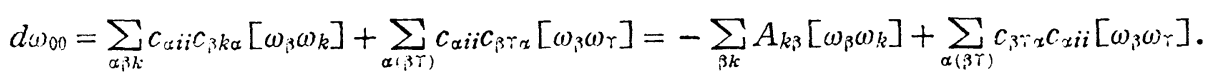

and we get $d \omega_{n}=\sum_{k}\left[\omega_{k} \omega_{k 0}\right]$ by virtue of (4.8).

Thus if $n>2$ and the relations

$$
c_{i j k}=0, \quad c_{i j \alpha}=0
$$




$$
c_{\alpha i j}=-c_{\alpha j i} \quad(i \neq j), \quad c_{\alpha i i}=c_{\alpha}
$$

are satisfied we get (1.11) by putting

$$
\pi_{i j}=-\sum_{\alpha} c_{\alpha i j} \omega_{\alpha}, \quad \omega_{00}=-\pi_{i i}=\sum_{\alpha} c_{\alpha} \omega_{\alpha}, \quad \omega_{i 0}=\sum_{\alpha \beta} c_{i \beta \alpha} c_{\alpha} \omega_{\beta} .
$$

In the case $n=2$ we must add one more condition (4.9). The case of dimension one reduces to theorem 3.2 .

Hence we get the following.

TheOREM 4.1 A homogeneous space of projective type whose dimension is greater than 2 and whose linear group of isotropy is conformal can be locally imbedded into a conformal space of the same dimension preserving the grouptheoretical structure. If the dimension of the space of projective type is 2 and the linear group of isotropy is conformal and (4.9) holds, the space can be imbelded into the conformal space of dimension 2.

Mathematical Institute, Nagoya University 